\title{
Some Computational Aspects of Discrete Orthonormal Moments
}

\author{
R. Mukundan, Senior Member, IEEE
}

\begin{abstract}
Discrete orthogonal moments have several computational advantages over continuous moments. However, when the moment order becomes large, discrete orthogonal moments (such as the Tchebichef moments) tend to exhibit numerical instabilities. This paper introduces the orthonormal version of Tchebichef moments, and analyzes some of their computational aspects. The recursive procedure used for polynomial evaluation can be suitably modified to reduce the accumulation of numerical errors. The proposed set of moments can be used for representing image shape features and for reconstructing an image from its moments with a high degree of accuracy.
\end{abstract}

Index Terms-Discrete orthogonal polynomials, image reconstruction, orthonormal moments, Tchebichef moments.

\section{INTRODUCTION}

$\mathbf{M}$ OMENT functions are used in several computer vision and related applications, such as pattern recognition, object identification, template matching, and pose estimation [1]-[4]. Zernike, Pseudo-Zernike, and Legendre moments use continuous orthogonal polynomials as basis functions and provide better feature representation capability and improved robustness to image noise over other types of moments [5]-[7]. Since the Zernike and Legendre polynomials are defined only inside the unit circle, the computation of those moments require a coordinate transformation and suitable approximation of the continuous moment integrals [8]-[11]. Discrete orthogonal moments such as the Tchebichef (Chebyshev) moments [12], [13] are directly defined in the image coordinate space and preserve the property of orthogonality in a moment set. Tchebichef moments are thus expected to perform better than continuous moments, particularly in applications requiring independent shape characteristics. The accuracy of image reconstruction with Tchebichef moments is distinctly better than with continuous orthogonal moments [14].

The scaled Tchebichef polynomials given in [12] provide a simple framework for generating the moment functions without significant variation in the range of magnitudes. However, when the moment order becomes large, the squared norm of the scaled Tchebichef polynomials assumes very small values, leading to numerical instabilities in the computed moments. This paper introduces the theoretical foundation for orthonormal moments with unit squared norm for the basis functions. This scheme also

Manuscript received February 3, 2003; revised December 10, 2003. The associate editor coordinating the review of this manuscript and approving it for publication was Dr. Thrasyvoulos N. Pappas.

The author is with the Department of Computer Science, University of Canterbury, Christchurch, New Zealand (e-mail: mukund@ cosc.canterbury.ac.nz).

Digital Object Identifier 10.1109/TIP.2004.828430 allows for renormalization of the moment functions whenever necessary, to nullify the effects of small truncation errors.

Another problem encountered in the computation of Tchebichef moments of large order is the propagation of numerical errors while using the recurrence relation for evaluating the polynomial values. The errors, thus, accumulated in the recursive procedure can have an exponential growth, rendering many high-order moment terms unusable. This phenomenon severely affects the quality of image reconstruction, particularly when large images (typically of size greater than $100 \times 100$ pixels) are used. A solution to the problem can be devised by suitably changing the recurrence relations to avoid cumulative multiplication of large values.

The organization of the paper is as follows. The next section reviews the key formulae for defining the scaled Tchebichef polynomials and the associated moments and illustrates the problems in image reconstruction using high-order moments. The orthonormal version of Tchebichef polynomials is introduced in Section III. Section IV presents ways to minimize the accumulation of truncation errors in moment computation. Some properties of orthonormal moments are discussed in Section V.

\section{TCHEBICHEF POLYNOMIALS AND MOMENTS}

For a given positive integer $N$ (usually the image size), and a value $x$ in the range $[0, N-1]$, the scaled Tchebichef polynomials $t_{n}(x), n=0,1, \ldots, N-1$, are defined using the following recurrence relation [12]:

$t_{n}(x)=\frac{(2 n-1) t_{1}(x) t_{n-1}(x)-(n-1)\left(1-\frac{(n-1)^{2}}{N^{2}}\right) t_{n-2}(x)}{n \quad n=2,3, \ldots N-1}$

where

$$
\begin{aligned}
& t_{0}(x)=1, \\
& t_{1}(x)=\frac{2 x+1-N}{N} .
\end{aligned}
$$

The above definition uses the following scale factor [12] for the polynomial of degree $n$

$$
\beta(n, N)=N^{n}
$$

The set $\left\{t_{i}\right\}$ has a squared-norm given by

$$
\begin{aligned}
\rho(n, N) & =\sum_{i=0}^{N-1}\left\{t_{i}(x)\right\}^{2} \\
& =\frac{N\left(1-\frac{1}{N^{2}}\right)\left(1-\frac{2^{2}}{N^{2}}\right) \cdots\left(1-\frac{n^{2}}{N^{2}}\right)}{2 n+1} .
\end{aligned}
$$


Squared Norm $(\mathbf{N}=\mathbf{2 0 0})$

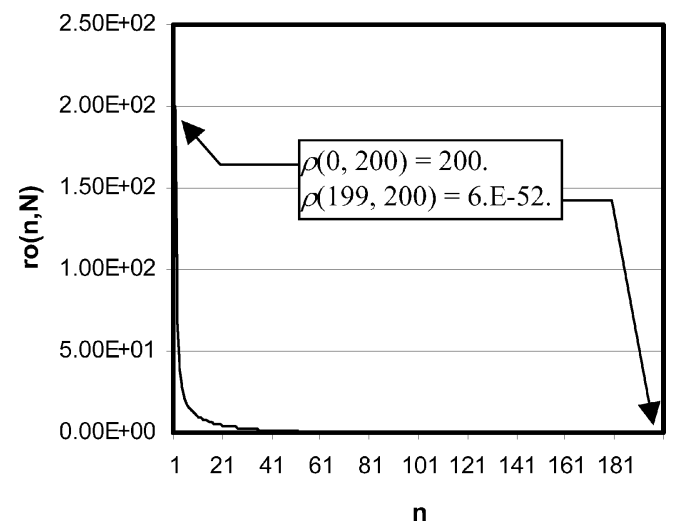

Fig. 1. Plot of the squared-norm of the scaled Tchebichef polynomials for $N=200$.

The scale factor in (3) was introduced to counteract the increase in magnitude of the polynomial values with the degree $n$. However, in situations where $N$ (the image size) is large and the polynomial degree approaches $N$, the squared-norm given in (4) can tend to a value close to zero. Fig. 1 shows a plot of $\rho(n, N)$ with $n$, for $N=200$.

The values of the squared-norm affect the magnitudes of the corresponding moments $T_{m n}$ of an image, as they are computed according to the following formula:

$$
\begin{aligned}
T_{m n}=\frac{1}{\rho(m, N) \rho(n, N)} \sum_{x=0}^{N-1} \sum_{y=0}^{N-1} t_{m}(x) t_{n}(y) f(x, y) \\
m, n=0,1,2, \ldots N-1
\end{aligned}
$$

where $f(x, y)$ denotes the intensity value at the pixel position $(x, y)$ in the image. Obviously, the computation of $T_{m n}$ can lead to erroneous results when $N$ is large. As an example, consider the process of reconstruction of an image from its moments, using the inverse moment transformation

$$
\widetilde{f}(x, y)=\sum_{m=0}^{M} \sum_{n=0}^{M} T_{m n} t_{m}(x) t_{n}(y)
$$

where $M$ denotes the maximum order of moments used and $\widetilde{f}(x, y)$ the reconstructed intensity distribution. For a binary image, the reconstruction error can be defined as

$$
\varepsilon=\sum_{i=0}^{N-1} \sum_{j=0}^{N-1}|\{f(i, j)-\tilde{f}(i, j)\}|
$$

and, for a gray-level image, as

$$
\varepsilon=\sqrt{\sum_{i=0}^{N-1} \sum_{j=0}^{N-1}\{f(i, j)-\tilde{f}(i, j)\}^{2}} .
$$

The images in Fig. 2 are used to illustrate the problems associated with large-order moments in image reconstruction. Fig. 3(a) and (b) show some of the reconstructions of image-1 as the maximum order of moments is increased from 10 to 95.
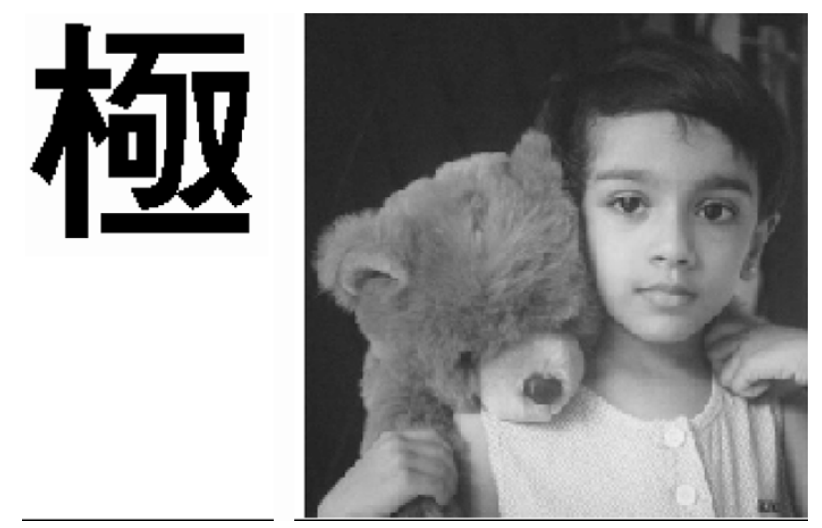

Fig. 2. Two images used for reconstruction. Image- 1 is a binary image of size $96 \times 96$ pixels and image- 2 is a gray-level image of size $200 \times 200$ pixels.

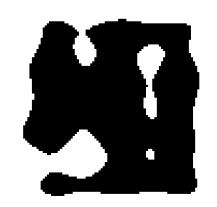

$M=10$

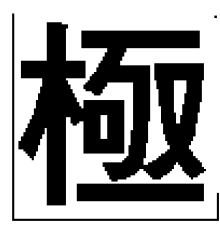

$M=85$

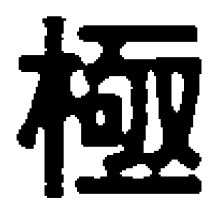

$M=30$

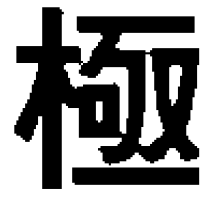

$M=50$

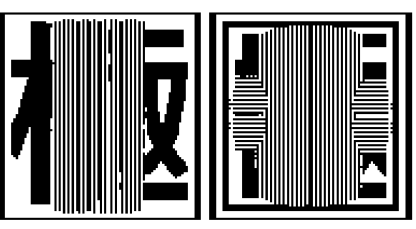

$M=95$
Fig. 3. (a) Improvement in the quality of image reconstruction as the maximum moment order $M$ is increased from 0 to 50. (b) Deterioration in the quality of image reconstruction as the maximum moment order $M$ is increased from 85 to 95 .

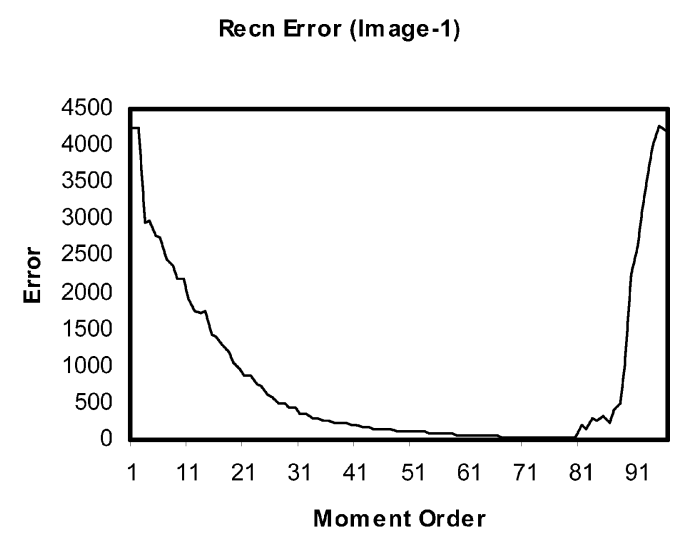

Fig. 4. Reconstruction error with respect to the maximum moment $\operatorname{order} M$, for image-1.

The reconstruction error for image-1, as computed from (7), clearly shows the region where the numerical errors start to dominate in higher-order moments (Fig. 4). The error starts to increase when $M=81$, toward the end (84\%) of the total range of values $(N=96)$. Similarly, the plot of the reconstruction error computed using (8) for image-2 also shows a region of numerical instability (Fig. 5) starting from $M=116$, which is at $58 \%$ of the total range of moments $(N=200)$. 


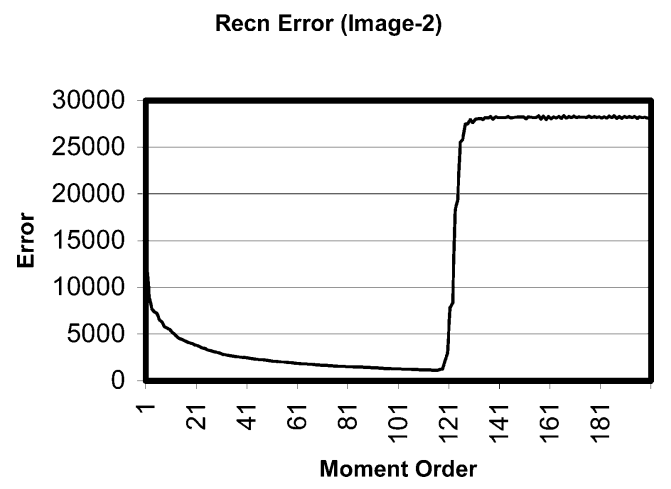

Fig. 5. Reconstruction error for image-2.

\section{ORTHONORMAL MOMENTS}

Toward the development a robust method for computing highorder discrete orthogonal moments, we will first attempt to solve the problem shown in Fig. 1, by constructing orthonormal versions of Tchebichef polynomials. This can be done by modifying the scale factor in (3) as

$$
\beta(n, N)=\sqrt{\frac{N\left(N^{2}-1\right)\left(N^{2}-2^{2}\right) \ldots\left(N^{2}-n^{2}\right)}{2 n+1}} .
$$

If we denote the new set of polynomials with the above scale factor, by $\left\{\hat{t}_{i}\right\}$, then it can be easily seen that the recurrence relations given in (1) now change to the following:

$$
\begin{array}{r}
\hat{t}_{n}(x)=\alpha_{1} x \hat{t}_{n-1}(x)+\alpha_{2} \hat{t}_{n-1}(x)+\alpha_{3} \hat{t}_{n-2}(x), \\
n=2,3, \ldots N-1 ; \quad x=0,1, \ldots N-1
\end{array}
$$

where

$$
\begin{aligned}
& \alpha_{1}=\frac{2}{n} \sqrt{\frac{4 n^{2}-1}{N^{2}-n^{2}}} \\
& \alpha_{2}=\frac{(1-N)}{n} \sqrt{\frac{4 n^{2}-1}{N^{2}-n^{2}}} \\
& \alpha_{3}=\frac{(n-1)}{n} \sqrt{\frac{2 n+1}{2 n-3}} \sqrt{\frac{N^{2}-(n-1)^{2}}{N^{2}-n^{2}}} .
\end{aligned}
$$

The starting values for the above recursion can be obtained from the following equations:

$$
\begin{aligned}
& \hat{t}_{0}(x)=\frac{1}{\sqrt{N}}, \\
& \hat{t}_{1}(x)=(2 x+1-N) \sqrt{\frac{3}{N\left(N^{2}-1\right)}} .
\end{aligned}
$$

\begin{tabular}{|c|c|c|c|c|c|}
\hline$n^{x}$ & 0 & 1 & 2 & .. & $N-1$ \\
\hline 0 & 11 & 1 & 1 & 1 & 1 \\
\hline 1 & 71 & 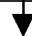 & \pm & 1 & $\frac{1}{7}$ \\
\hline 2 & 1 & & & & \\
\hline & $\nabla$ & $\rightarrow$ & & & \\
\hline$N-1$ & & & & & \\
\hline
\end{tabular}

We denote the squared norm by $\hat{\rho}(n, N)$, so that

$$
\hat{\rho}(n, N)=\sum_{i=0}^{N-1}\left\{\hat{t}_{n}(i)\right\}^{2}=1.0 .
$$

The moment equations in (5) now reduce to

$$
\begin{aligned}
& T_{m n}=\sum_{x=0}^{N-1} \sum_{y=0}^{N-1} \hat{t}_{m}(x) \hat{t}_{n}(y) f(x, y), \\
& m, n=0,1,2, \ldots N-1
\end{aligned}
$$

Fig. 6. $N \times N$ matrix represents the set of orthonormal polynomial values, with the solid and the dotted arrows denoting $n$ recursion and $x$ recursion respectively.

while the inverse moment transform becomes

$$
\begin{aligned}
\tilde{f}(x, y) & =\sum_{m=0}^{M} \sum_{n=0}^{M} T_{m n} \hat{t}_{m}(x) \hat{t}_{n}(y) . \\
x, y & =0,1,2, \ldots N-1 .
\end{aligned}
$$

Since the new moment set is orthonormal, small errors in the numerical values of the Tchebichef polynomials can be corrected using renormalization

$$
\hat{t}_{n}(x) \leftarrow \frac{\hat{t}_{n}(x)}{\sqrt{\sum_{i=0}^{N-1}\left\{\hat{t}_{i}(i)\right\}^{2}}}, \quad x=0,1,2, \ldots N-1 .
$$

However, systems where numerical errors are recursively propagated in each step cannot be corrected using renormalization alone. Indeed, the use of orthonormal moments with renormalization of high-order moments has only marginally improved the results presented earlier in Figs. 4 and 5.

\section{RECURRENCE RELATIONS}

Note that $\hat{t}_{n}(x)$ is a polynomial in $x^{n}$, and, therefore, the term $\hat{t}_{N-1}(N-1)$ for $N=200$ should, at least in theory, contain the term $200^{200}$ multiplied by an infinitely small coefficient so that the magnitude is less 1 . Numerical instability can therefore easily occur in the evaluation of such polynomials if the recurrence relations are not properly used. A closer look at (10) and (11) will tell us than none of the coefficients $\alpha_{1}, \alpha_{2}, \alpha_{3}$ is large, and the only factor that contributes to large magnitude variations is the repeated multiplication by $x$. The $n$ recursion given in (10) is represented by arrows with solid lines in Fig. 6. We will use this recurrence relation only to compute the polynomial values for $x=0$ and then fill each row of table in Fig. 6 using the $x$ recursion given below.

We know that [15] and [16]

$$
\hat{t}_{n}(0)=\frac{(1-N)(2-N) \ldots(n-N)}{\beta(n, N)}, \quad n=0,1 \ldots N-1 .
$$

and using (9), we get

$$
\hat{t}_{n}(0)=-\sqrt{\frac{N-n}{N+n}} \sqrt{\frac{2 n+1}{2 n-1}} \hat{t}_{n-1}(0), \quad n=1, \ldots N-1 .
$$



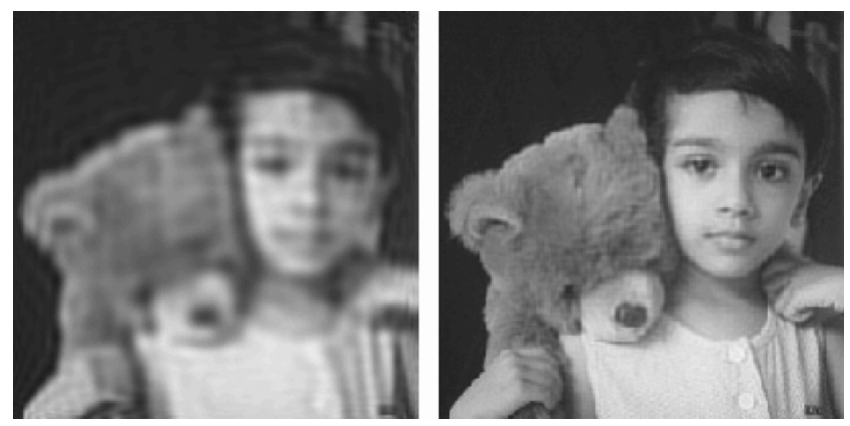

Fig. 7. Two reconstructions of image-2, using a maximum moment order of 65 and 199 , respectively $(N=200)$.

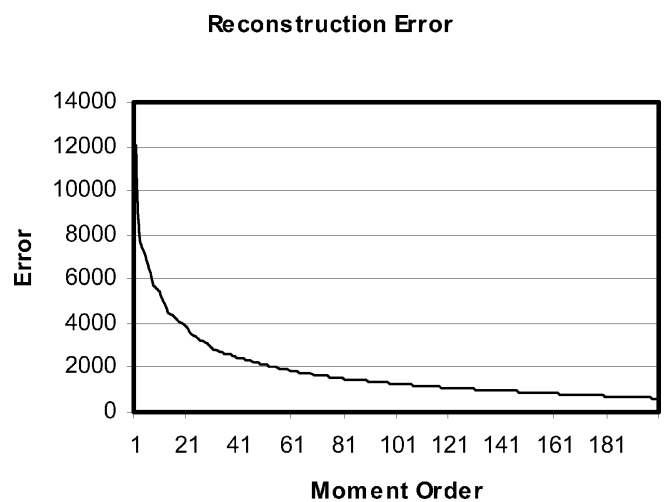

Fig. 8. Plot of image-2 reconstruction error with moments computed using (18)-(22).

Also, we have [15]

$$
\hat{t}_{n}(1)=\left\{1+\frac{n(1+n)}{1-N}\right\} \hat{t}_{n}(0) .
$$

Equations (18) and (19) can be used to obtain the starting values for the following $x$ recursion:

$$
\begin{array}{r}
\hat{t}_{n}(x)=\gamma_{1} \hat{t}_{n}(x-1)+\gamma_{2} \hat{t}_{n}(x-2), \\
n=1,2, \ldots N-1 ; \quad x=2,3, \ldots \frac{N}{2}
\end{array}
$$

where

$$
\begin{aligned}
\gamma_{1} & =\frac{-n(n+1)-(2 x-1)(x-N-1)-x}{x(N-x)} \\
\gamma_{2} & =\frac{(x-1)(x-N-1)}{x(N-x)} .
\end{aligned}
$$

Also note that in (20), the recursion can be terminated at $x=$ $N / 2$, since we can make use of the symmetry condition

$$
\hat{t}_{n}(N-1-x)=(-1)^{n} \hat{t}_{n}(x)
$$

to evaluate the polynomial values where $x$ is in the range $[N / 2$, $N-1]$. This greatly reduces the computational time, and the amount of accumulated errors in the result. Equation (20) was

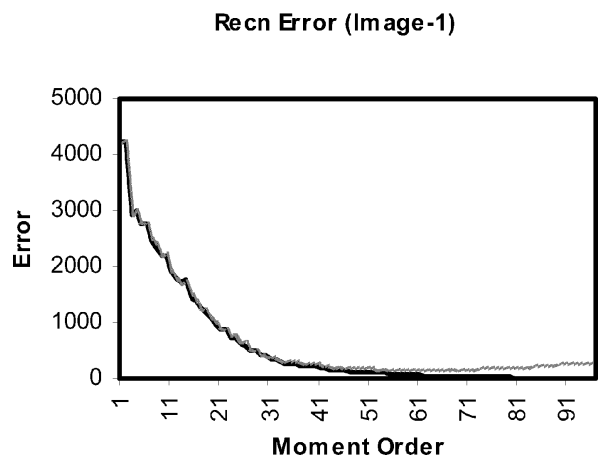

Fig. 9. Plot of image-1 reconstruction error with moments computed using (18)-(22). The dotted line shows the change in error when noise is added to the input image.

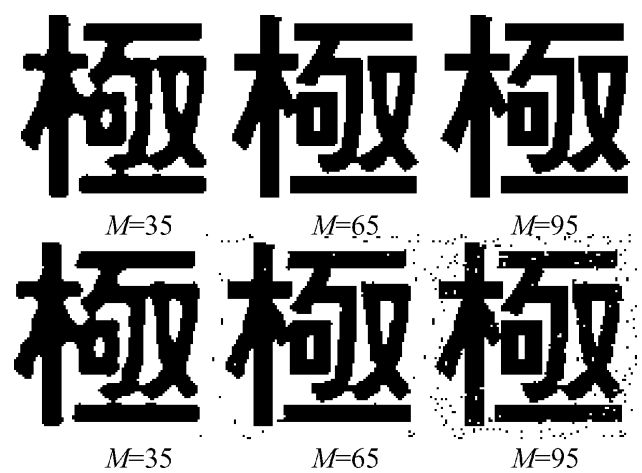

Fig. 10. (a) Reconstruction of image-1 without noise, using $x$ recursion. $(N=$ $96)$. (b) Reconstruction of image-1 with 5\% noise, using $x$ recursion. $(N=96)$.

used to compute the moments of image-2 (Fig. 2) and a few reconstructions employing high-order moments are shown in Fig. 7.

Comparing the reconstruction error in Fig. 8 with that given in Fig. 5, we find that the method using $x$ recursion provides a significantly high level of stability and accuracy in the computed high-order moments. The results of the analysis with image-1 are in Fig. 9 (compare this with Fig. 4). The dotted line indicates the reconstruction error after adding 5\% salt-and-pepper noise to the binary image. A few reconstructions of image- 1 with and without noise added are shown in Fig. 10(a) and (b).

\section{A FEW Properties of ORTHONORMAL POLYNOMIALS}

Since $\hat{t}_{0}(x)$ is a constant polynomial, the orthogonality property implies

$$
S_{0}=\sum_{x=0}^{N-1} \hat{t}_{n}(x)=0, \quad \text { for all } n>0 .
$$

In general

$$
S_{\mathrm{k}}=\sum_{x=0}^{N-1} x^{k} \hat{t}_{n}(x)=0, \quad \text { for all } n>k .
$$

The above equations can be used as additional constraints on the polynomial table (Fig. 6) to improve the accuracy of the computed values. Using (10) and the orthonormal properties of 
$\hat{t}_{i}(x)$, we can express the coefficients $\alpha_{1}, \alpha_{2}, \alpha_{3}$ as sums of products of the polynomial values

$$
\begin{aligned}
\alpha_{1} & =\frac{1.0}{\sum_{x=0}^{N-1} x \hat{t}_{n}(x) \hat{t}_{n-1}(x)} \\
\alpha_{2} & =-\alpha_{1} \sum_{x=0}^{N-1} x\left\{\hat{t}_{n-1}(x)\right\}^{2} \\
\alpha_{3} & =-\alpha_{1} \sum_{x=0}^{N-1} x \hat{t}_{n-1}(x) \hat{t}_{n-2}(x) .
\end{aligned}
$$

The symmetry property of the Tchebichef polynomials (22) can be used to simplify (13) as

$$
\hat{\rho}(n, N)=2 \sum_{i=0}^{N / 2}\left\{\hat{t}_{n}(i)\right\}^{2}=1.0, \quad \text { when } N \text { is even. }
$$

From (17), we can derive a simple expression for $\hat{t}_{N-1}(0)$ as

$$
\hat{t}_{N-1}(0)=(-1)^{N-1} \prod_{\mathrm{k}=1}^{N-1} \sqrt{\frac{k}{N+k-1}}
$$

which can used to start the recursive computation in (18) in the reverse direction (with decreasing values of $n$ ).

\section{CONCLUSION}

Discrete orthogonal moments provide several advantages over continuous moments such as the Zernike and the Legendre moments. The elimination of numerical approximations and coordinate transformations in the computation of discrete moments are very useful in preserving the orthogonality properties in a moment set. However, the recursive nature of polynomial evaluation can lead to numerical problems when the required moment order is large.

This paper has introduced the theoretical framework for the orthonormal version of Tchebichef polynomials. Orthonormalization eliminates one region of numerical instability while computing high-order moments, but recurrence relations can still induce large errors as the moment order increases. The paper has also proposed using recursion along the $x$-direction (coordinate values) rather than the $n$-direction (moment order) to minimize the propagation of numerical errors. Image reconstruction using orthogonal moments has been used as an example to illustrate the stability of the results with the proposed method.

\section{REFERENCES}

[1] A. Khotanzad, "Invariant image recognition by Zernike moments," IEEE Trans. Pattern Anal. Mach. Intell., vol. 12, pp. 489-497, Mar. 1990.
[2] S. X. Liao and M. Pawlak, "On image analysis by moments," IEEE Trans. Pattern Anal. Mach. Intell., vol. 18, pp. 254-266, Feb. 1996.

[3] M. R. Teague, "Image analysis via the general theory of moments," $J$. Optical Soc. Amer, vol. 70, pp. 920-930, 1980.

[4] R. Mukundan, "Estimation of quaternion parameters from two-dimensional image moments," Graph. Models Image Process., vol. 54, pp 345-350, 1992.

[5] S. O. Belkasim, "Pattern recognition with moment invariants - A comparative study and new results," Pattern Recognit., vol. 24, pp. $1117-1138,1991$.

[6] A. Wallin, "Complete sets of complex Zernike moment invariants and the role of pseudo-invariants," IEEE Trans. Pattern Anal. Mach. Intell., vol. 17, pp. 1106-1110, Nov. 1995.

[7] S. O. Belkasim, M. Ahmadi, and M. Sridhar, "An efficient algorithm for fast computation of Zernike moments," in IEEE 39th Midwest Symp. Circuits and Systems, vol. 3, 1996, pp. 1401-1404.

[8] R. Mukundan and K. R. Ramakrishnan, Moment Functions in Image Analysis - Theory and Applications. Singapore: World Scientific, 1998.

[9] - "Fast computation of legendre and Zernike moments," Pattern Recognit., vol. 28, pp. 1433-1442, 1995.

[10] C. W. Chong, P. Raveendran, and R. Mukundan, "A comparative analysis of algorithms for fast computation of Zernike moments," Pattern Recognit., vol. 36, pp. 731-742, 2003.

[11] — "Translation invariants of Zernike moments," Pattern Recognit., vol. 36, no. 8, pp. 1765-1773, 2003.

[12] R. Mukundan, S. H. Ong, and P. A. Lee, "Image analysis by Tchebichef moments," IEEE Trans. Image Processing, vol. 10, pp. 1357-1364, Sept. 2001.

[13] — - "Discrete orthogonal moment features using Chebyshev polynomials," in Proc. Image Vision Computing New Zealand , 2000, pp. 20-25.

[14] — "Discrete vs. continuous orthogonal moments for image analysis," in Proc. Intl. Conf. Imaging Science Systems and Technology, vol. 1, 2001, pp. 23-29.

[15] A. Erdelyi et al., Higher Transcendental Functions. New York: McGraw Hill, 1953, vol. 2.

[16] A. V. Nikiforov, S. K. Suslov, and V. B. Uvarov, Classical Orthogonal Polynomials of a Discrete Variable. New York: Springer-Verlag, 1991.

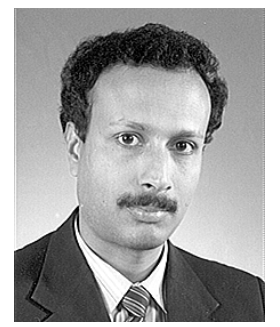

R. Mukundan (SM'96) received the Ph.D degree from the Indian Institute of Science, Bangalore, in 1996, for his research work on "image based attitude and position estimation using moment functions."

He was a Senior Scientist with the Control Systems Group, Indian Space Research Organization (ISRO) Satellite Centre, Bangalore, from 1982 to 1997. Later, he joined the Faculty of Information Science and Technology at Multimedia University (MMU), Melaka, Malaysia, where he held the positions of Associate Professor and the Chairman of the Centre for Mathematical Modeling and Computational Science until December 2001. Currently, he is a Senior Lecturer in the Department of Computer Science, University of Canterbury, Christchurch, New Zealand. He has authored a research monograph titled Moment Functions in Image Analysis - Theory and Applications (Singapore: World Scientific, 1998). $\mathrm{He}$ has also authored a book titled Computer Graphics Algorithms in Java (Malaysia: Prentice-Hall, 2000). His primary research interests are in the areas of moment-based feature descriptors and their applications in computer vision, computer graphics algorithms, and image-based rendering. 